\title{
Caracterización de la Contaminación Atmosférica por Calcinación de Yeso en los Municipios de Araripina y Trindade, Noreste de Brasil
}

\section{Characterization of air Pollution by Calcination of Gypsum in the Municipalities of Araripina and Trindade, Northeast of Brazil}

\author{
${ }^{a}$ Universidad Internacional Iberoamericana. México. \\ 'Instituto Politécnico Nacional. México. \\ *E-mail: rflorencio@ipn.mx
}

Givanilda Honório Silvaa; Miguel Ysrrael Ramírez-Sánchezª Rodrigo Florencio da Silva*b

\begin{abstract}
Resumen
Este manuscrito presenta a través de un estudio tipo no experiemental la caracterización de la contaminación atmosférica por la calcinación de yeso en los municipios de Araripina y Trindade del estado de Pernambuco, en el noreste de Brasil. Esos municipios son importantes por conformar el Complejo Yesero de Araripe que representa la mayor parte de la producción de yeso de Brasil, considerada una de las mayores del mundo. La investigación se realizó utilizando datos de informes de pruebas de caracterización de emisiones atmosféricas realizadas en 20 empresas, 9 de Trindade y 11 de Araripina, de 2015 a 2018. Según los resultados, no hubo una reducción significativa en las emisiones de contaminantes entre 2015 y 2016, lo que evidencia pocas inversiones en el mantenimiento y mejora de los procesos. Sin embargo, para el período 2017-2018, los datos muestran una disminución en las emisiones de contaminantes, con un número considerable de empresas que cumplen con los límites establecidos por la ley. Dado lo anterior, existe la necesidad de intensificar la supervisión de las empresas de yeso en la región debido los hornos se encuentran en condiciones precarias sin mantenimiento y mejora en el proceso de combustión donde las emisiones de monóxido de carbono y otros contaminantes que exceden en mucho el límite permitido por la legislación ambiental.
\end{abstract}

Palabras-clave: Contaminación del aire, Residuos de yeso, Análisis isocinético.

\begin{abstract}
This manuscript presents through a non-experimental type study, the characterization of air pollution by the calcination of plaster in the municipalities of Araripina and Trindade in the state of Pernambuco, in northeastern Brazil. These municipalities are important for shaping the Plaster Complex of Araripe, which represents the majority of the plaster production in Brazil, considered one of the largest in the world. The research was conducted using data from reports of atmospheric emissions characterization tests carried out in 20 companies, 9 from Trindade and 11 from Araripina, from 2015 to 2018. According to the results, there was no significant reduction in pollutant emissions between 2015 and 2016, which shows few investments in the maintenance and improvement of the processes. However, for the 2017-2018 period, the data shows a decrease in pollutant emissions, with a considerable number of companies that meet the limits established by law. Given the above, there is a need to intensify the supervision of gypsum companies in the region because the furnaces are in precarious conditions without maintenance and improvement in the combustion process where emissions of carbon monoxide and other pollutants that greatly exceed the limit allowed by environmental legislation.
\end{abstract}

Keywords: Atmospheric pollution, Plaster waste, Isokinetic analysis.

\section{Introducción}

El desarrollo tecnológico experimentado por la industria en las últimas décadas es un reflejo del capital económico y humano que se ha empleado desde los primeros días de la Revolución Industrial (GONÇALVES; NASCIMENTO, 2011). Estos desarrollos pueden ser fácilmente percibidos en varios sectores de la industria y entre ellos uno de gran importancia es la construcción civil (BARBISAN et al., 2011).

La industria de la construcción se destaca como uno de los principales ejes de la economía de una nación y es responsable de gran parte del Producto Interno Bruto (PIB) cuando se trata de los países en desarrollo, ya que tiene una enorme capacidad para absorber la mano de obra poco calificada de ese territorio, como sucede en Brasil (CBIC, 2018).

A pesar de su importancia en el desarrollo económico de un país, la construcción civil también se ha vinculada a varios problemas, especialmente cuando se trata de los efectos negativos sobre el medio ambiente (BARBISAN et al., 2011). Esta industria es responsable de entre el $40 \%$ y el $75 \%$ de toda la materia prima que se consume en el mundo, es decir, también termina impactando el medio ambiente con la enorme generación de residuos y contaminación ligada a este proceso (GASQUES, 2014).

Según John y Cincotto (2003), además de esta enorme carga de impactos ambientales vinculados al proceso de extracción de materiales, la construcción también tendrá un impacto en el desarrollo de sus actividades constructivas, con diferentes barreras vinculadas a cada tipo de material utilizado en la construcción.

En los últimos años un material que ha ido ganando mucha importancia tanto en el medio técnico como en el académico es el yeso, un producto derivado del mineral gipsita. Este 
crecimiento en su uso se debe a las diversas características intrínsecas del material y a las ventajas económicas de su extracción y manejo (JOHN; CINCOTTO, 2003).

Entre los problemas ambientales vinculados a los procesos que envuelven al yeso cabe mencionar la gestión de sus residuos y las posibles emisiones gaseosas que involucran su proceso productivo (PINHEIRO, 2011). El yeso es un tipo de material que actúa como aglutinante hidrófilo y se produce básicamente mediante el proceso de calcinación del mineral gipsita, sobre una presión atmosférica en hornos industriales con temperaturas que oscilan entre 135 y 180 grados centígrados (LUSHNIKOVA; DVORKIN, 2016).

La calcinación de la gipsita puede traducirse como el proceso de eliminación de agua, es decir, la deshidratación del agua que, cuando se produce, libera a la atmósfera varios componentes contaminantes, incluido el dióxido de carbono (LUSHNIKOVA; DVORKIN, 2016).

Las ciudades que conforman el Complejo Yesero de Araripe, que representa más del $90 \%$ de toda la producción de yeso en Brasil (actualmente el undécimo mayor productor de yeso del mundo), experimentan de cerca los efectos negativos sobre el medio ambiente causados por esta industria (MEDEIROS, 2003).

Para comprender mejor los efectos nocivos asociados con las emisiones gaseosas que ocurren durante la calcinación del yeso en el Complejo Yesero de Araripe, específicamente en las ciudades de Araripina y Trindade, se identificó la emisión atmosférica en esta región a través de la caracterización de sus contaminantes analizando la cantidad de partículas y gases presentes.

\section{Material y Métodos}

La investigación realizada fue de tipo no experimental, utilizando datos ya producidos por el Instituto SENAI de Tecnología en Medio Ambiente, con una serie de momentos o puntos cronológicos en los que se recogieron los datos. La modalidad fue longitudinal en la que permite analizar el cambio de una o más variables y sus relaciones.

La variable dependiente utilizada fue el combustible utilizado en los hornos de la industria de calcinación, ya que dependiendo del combustible, la emisión de contaminantes puede ser mayor o menor. Los tipos de contaminantes también varían según el combustible o los tipos de gases emitidos a la atmósfera. La investigación se llevó a cabo mediante la realización de pruebas de caracterización química de veinte empresas centradas en la calcinación de yeso en los municipios de Araripina y Trindade, de 2015 a 2018.

El alcance geográfico está definido en el título de la investigación y el proyecto es un estudio de caso múltiple en la región de Araripe del Nordeste brasileño, en calcinadores de yeso.

\subsection{Histórico de la Contaminación Atmosférica}

El aire respirable es uno de los mayores dones de la naturaleza, y junto con el agua es uno de los principales insumos para el desarrollo de los seres vivos en la tierra. Jardim (2001) comenta que el aire que compone nuestra atmósfera comenzó su formación hace unos 65 millones de años, estando compuesto básicamente por la mezcla de varios gases entre ellos los necesarios para sostener la vida en la tierra.

La composición de esta masa de aire está formada básicamente por: $78 \%$ nitrógeno, $21 \%$ oxígeno y $1 \%$ otros gases, como el dióxido de carbono o el vapor de agua. Al realizar el acto de respirar un individuo básicamente realiza este intercambio de gases con la naturaleza, absorbiendo sólo oxígeno y expulsando dióxido de carbono junto con los demás gases absorbidos que no contribuirán al mecanismo de la respiración (JARDIM, 2001).

De los gases que componen la atmósfera terrestre se da gran importancia al oxígeno que, como se ha mencionado anteriormente, es el principal gas utilizado en la respiración de los seres vivos, siendo esencial para asegurar su calidad para el correcto desarrollo de las especies terrestres. Según Mouvier (1995), el aire que se consideraría "puro", es decir, totalmente formado por oxígeno y nitrógeno, no existe en la naturaleza, principalmente debido a la interferencia que sufre el medio ambiente natural como resultado tanto de procesos naturales como de agentes externos o antrópicos.

Las interferencias en la atmósfera causadas por el propio entorno natural pueden producirse de varias maneras, como el levantamiento de partículas de polvo mediante tormentas de viento, que en este caso servirían de mecanismo de transporte, y las nubes de humo resultantes de la actividad de los volcanes que, debido a la temperatura y al peso de las partículas de ceniza, pueden ser transportadas por kilómetros (MOUVIER, 1995).

El desarrollo de las civilizaciones y la mejora de los procesos productivos e industrializados que tuvieron lugar junto con el avance tecnológico, desencadenado principalmente durante la revolución industrial, fue uno de los hitos para el inicio de la interferencia antrópica con el medio ambiente natural que se refleja principalmente en la atmósfera (GONÇALVES; NASCIMENTO, 2011).

El mecanismo de contaminación atmosférica ocurre con la exposición continua del aire a la contaminación que proviene de las más variadas fuentes contaminadora. Los agentes contaminantes se traducen en los más diversos agentes, entre ellos los óxidos de nitrógeno, las partículas compuestas de varios contaminantes, tanto líquidos como sólidos, los compuestos de azufre y el monóxido de carbono (SANTOS, 2006).

Ciudades como Londres, que experimentaron un crecimiento acelerado durante la Revolución Industrial, 
sufrieron mucho por la contaminación generada por fábricas y vehículos con motores de combustión (GONÇALVES; NASCIMENTO, 2011).

En el Complejo Yesero de Araripe, una muy alta concentración de fábricas y calcinadoras de yeso, han contribuido en gran medida al aumento de la generación de gas a partir de esta quema de material y a la presencia de partículas de polvo (MEDEIROS; HURTADO-GUERRERO; SILVA, 2010). La Tabla 1 muestra el número de industrias en este sector para el año 1995.

Tabla 1 - Distribución espacial de la industria yesera en el Complejo Yesero de Araripina - 1995.

\begin{tabular}{|l|c|c|c|c|c|}
\hline \multirow{2}{*}{$\begin{array}{c}\text { Ramos de la Cadena de } \\
\text { Producción de Yeso }\end{array}$} & \multicolumn{5}{c|}{ Unidades de Producción } \\
\cline { 2 - 6 } & Araripina & Ipubi & Trindade & Ouricuri & Bodocó \\
\hline Minas activas & 12 & 16 & 1 & 6 & 4 \\
\hline Calcinadoras & 18 & 7 & 20 & 2 & 0 \\
\hline Fábricas de Pre-moldeado & 74 & 26 & 13 & 7 & 5 \\
\hline Total & 104 & 49 & 34 & 15 & 9 \\
\hline
\end{tabular}

Fuente: Adaptado de Santos y Sardou (1996).

De acuerdo con la Oficina Internacional del Trabajo - OIT (1989), la acción de estas partículas de polvo y gases de la calcinación del yeso puede causar irritación de las mucosas del tracto respiratorio y de los ojos, provocando enfermedades como conjuntivitis, laringitis, entre otras.

Según Santos y Sardou (1996), Araripina es el municipio con mayor actividad industrial del Complejo Yesero, albergando a buena parte de su población en actividades relacionadas con el sector, se estima que alrededor del $47 \%$ del total de los empleos generados en la región para el año 1996 han sido generados por esta industria, lo que atestigua la cantidad de personas que se ven directamente afectadas por la contaminación causada por ella.

\subsection{Calidad del aire}

La contaminación del aire puede definirse por la presencia de materiales o formas de energía en el aire que aumentan el riesgo de daños y/o molestias de carácter grave para las personas y el medio ambiente natural (GALAN et al., 2003).

Los procesos de dispersión de contaminantes en la atmósfera por partículas minerales se observan con frecuencia en calcinadores y se limitan básicamente a la proximidad de la fuente de emisión, que representa un área muy extensa de las ciudades de Araripina y Trindade (MEDEIROS; HURTADOGUERRERO; SILVA, 2010).

Fellenberg (1997) en su estudio sobre la industria de la caliza logró identificar precipitaciones de 1,74 gramos por metro cuadrado de polvo de caliza en vinaza de hasta $1 \mathrm{~km}$ y 0,27 gramos por metro cuadrado de polvo a una distancia de $2 \mathrm{~km}$, el rango de acción de esta partícula se determina de acuerdo al mecanismo de transporte, en este caso los vientos, hay casos en los que el polvo se percibe a una distancia de hasta $8 \mathrm{~km}$ de la fuente generadora, o los Hornos de Calcinación mostrados en la Figura 1.
Figura 1 - Horno de calcinación de yeso en la fábrica del interior de Araripe.

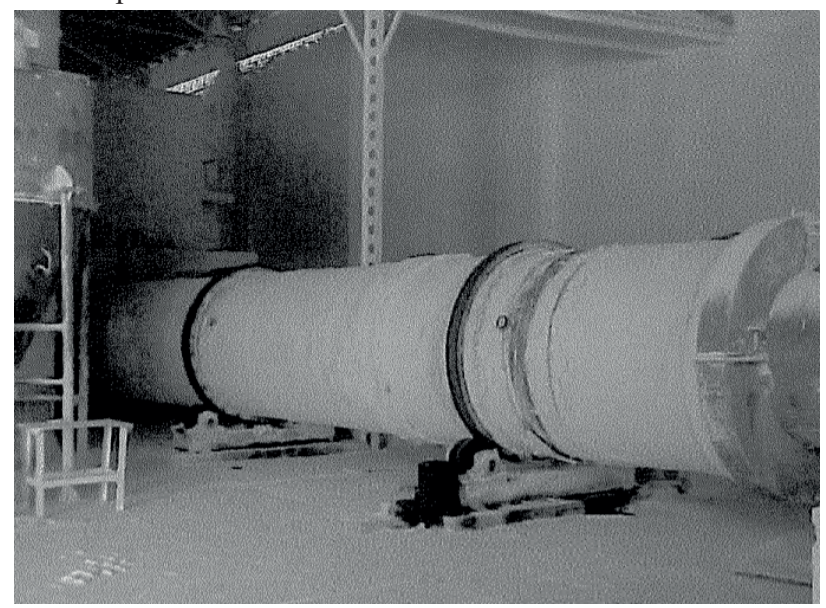

Fuente: Parente (2001).

En el medio ambiente que está directamente expuesto a estos agentes contaminantes es importante conocer sus características toxicológicas, ya que determinará las formas en que afectará al organismo/medio ambiente expuesto a sus efectos (BRASIL, 2001).

El yeso y todos los productos que forman parte de su proceso de producción son la principal actividad económica y el mayor empleador de la región que incluye a las ciudades del Centro Yesero de Araripe, un cambio drástico en el modelo de producción o sanciones legislativas rápidas para resolver el problema, aunque fuera a favor de mitigar los efectos nocivos para el medio ambiente y la población, podría causar daños irreversibles a la economía y aumentar el desempleo en la región (MEDEIROS; HURTADO-GUERRERO; SILVA, 2010).

Por lo tanto, para ayudar en la toma de decisiones de las entidades públicas y mejorar la gestión de la calidad del aire es seguramente necesario un análisis de caracterización de contaminantes y partículas liberadas en el proceso de calcinación del yeso, ya que con base en este análisis se podría determinar un escenario que simularía de una manera muy real los riesgos que enfrenta la población de estas ciudades 
(SANTOS, 2006).

\subsection{Principales contaminantes}

Entre las principales fuentes de estos agentes contaminantes se pueden destacar los siguientes:

- Contaminación por partículas

De acuerdo con la Resolución 382 de 2006 de CONAMA, que establece los límites máximos de emisión de contaminantes atmosféricos para fuentes fijas, se define a la materia particulada-MP como cualquier material sólido o líquido en una mezcla gaseosa, que permanece en este estado a temperatura ambiente.

La contaminación por partículas o Material Particulado (PM) puede abarcar varios contaminantes, tanto sólidos como líquidos, que pueden ser suspendidos en la atmósfera debido a su reducido tamaño (YANAGI; ACTION; BARROZO, 2012).

El PM está formado por partículas sólidas y/o líquidas, con excepción del agua, que entran en la atmósfera por vías naturales o, por la acción del hombre, el PM es un contaminante atmosférico asociado a efectos adversos para la salud humana (BRAGA et al., 2001; DOCKERY et al., 1993). La contaminación de los humanos ocurre a través de la respiración y el contacto con la piel, mediante la inhalación de partículas que pueden contaminar los pulmones o el tracto digestivo (SEVERO 2017).

Estas partículas pueden ser clasificadas de acuerdo a su tamaño, mecanismo de formación, origen, composición química, comportamiento atmosférico y método de medición (USEPA, 2012).

El material particulado puede estar formada por cuatro procesos diferentes: fricción o dispersión mecánica, combustión, nucleación homogénea y heterogénea y evaporación de gotas (SANTOS, 2006).

En el caso de la combustión, hay oxidación o vaporización de las sustancias orgánicas presentes en ellas, por lo tanto, las moléculas de combustible se reducen a cenizas y restos de combustión lenta, estas cenizas y restos estarán en un rango de 1 a $100 \mu \mathrm{m}$ de diámetro, constituyendo las partículas resultantes de este proceso de combustión (MEDEIROS, 2003).

Además de los daños a la salud humana, las partículas causan daños a la agricultura; daños a la vegetación y al suelo; contaminación por metales pesados (GURGEL; GIL, 2009).

- Monóxido de carbono

Es un gas incoloro y venenoso que se origina a partir del producto de los combustibles fósiles, emitido diariamente a la atmósfera en cientos de millones de toneladas, procedente de procesos naturales y antropogénicos (DRUMM et al., 2014).

Es el resultado de la quema de combustibles fósiles, el monóxido de carbono (CO), tiene afinidad por la hemoglobina, 240 veces mayor que el oxígeno, pudiendo saturar rápidamente la sangre y reducir la capacidad de la sangre para transportar oxígeno (CANÇADO et al., 2006). El CO es capaz de inhibir el intercambio de oxígeno en la sangre y en altas concentraciones puede causar la muerte por envenenamiento (PROCKOP; CHICHKOVA, 2007).

- Compuestos de azufre

El azufre presente en la atmósfera provoca impactos ambientales (deterioro de ecosistemas, contaminación de lechos, entre otros), así como cambios directos en el medio urbano, siendo estos cambios climáticos y daños a la salud de diversos organismos (CASTRO et al., 2013).

Los estudios de Eisinger y Burrows (1998) demuestran las relaciones del dióxido de azufre (SO2) y las variaciones en relación a los parámetros meteorológicos, el SO2 entra a la atmósfera por acciones antrópicas o naturales, muchas de estas emisiones provienen de la combustión de combustibles fósiles, siendo éste uno de los materiales precursores de la lluvia ácida.

Los compuestos de azufre pueden causar daños al medio ambiente natural y al suelo expuesto, además de la lluvia ácida antes mencionada, que a menudo causan efectos nocivos directos o indirectos en la salud humana, como irritación de la piel, ojos y mucosas, reacciones alérgicas, problemas dentales, cambios metabólicos, problemas del sistema respiratorio y daños en el sistema inmunológico (GURGEL et al., 2009).

\subsection{Estudio de caso: los municipios de Arapina y Trindade - Pernambuco}

El proyecto tiene un alcance ambiental, a través de la investigación de contaminantes atmosféricos en los calcinadores de yeso de Araripe, con resultados que promueven directrices de beneficios ambientales para la región. Miraglia y Gouveia (2014) describen que la contaminación del aire y las emisiones que afectan las cualidades del aire local pueden tener una interferencia global en términos del efecto invernadero y el cambio climático.

La variable dependiente utilizada fue el combustible utilizado en los hornos de las industrias de calcinación, ya que, dependiendo del combustible, la emisión de contaminantes puede ser mayor o menor. Los tipos de contaminantes también varían dependiendo del combustible. Tipos de gases emitidos a la atmósfera.

La investigación se llevó a cabo a través de pruebas de caracterización química de veinte empresas centradas en la calcinación de yeso en los municipios de Araripina y Trindade en el período de 2015 a 2018. Los datos recogidos forman un estudio de caso que implica un conocimiento detallado del objetivo del estudio, los calcinadores de yeso Araripe.

Las pruebas de medición de contaminantes atmosféricos fueron realizadas por equipos CIPA (Isokinetic Collection of Atmospheric Pollutants), el analizador de gases de combustión mediante la recolección del filtro de las chimeneas, siguió las siguientes Normas para el desarrollo de mediciones de gases y material particulado en las chimeneas de la ABNT NBR 10701/1989 (Fija las condiciones requeridas para la determinación y ubicación de los puntos de muestreo o la velocidad de toma de una sección transversal de los 
conductos y chimeneas a partir de fuentes fijas); ABNT NBR 11966/1989 (Método para determinar la velocidad promedio y el flujo volumétrico del efluente gaseoso en ducto o chimenea de fuente estacionaria, a través del tubo Pitot S); ABNT NBR 11967/1989 (método para determinar la humedad de los efluentes gaseosos en ductos y chimeneas de fuentes estacionarias); EPA 625/6-78-004 (USEPA, 1978, Guía para el control de la Agencia de Contaminación del Aire y la transferencia de tecnología de la protección ambiental).

Se llevó a cabo una encuesta en las industrias que tomaron muestras de contaminantes del aire entre 2015 y 2018, y el tipo de muestreo fue aleatorio simple.

La recolección de datos se realizó mediante investigación documental, utilizando los informes de prueba de mediciones de contaminantes atmosféricos realizados por el Instituto de Tecnología Ambiental del SENAI, estos informes de prueba contienen los contaminantes cuantitativos (CO, CO2, NOx, SOx y material particulado) que las industrias liberan a la atmósfera donde se recogieron todos los datos necesarios y se realizó un diagnóstico en relación con la contaminación del aire en los municipios de Trindade y Araripina en el interior de Pernambuco.

La caracterización de las emisiones de los hornos utilizados siguió el procedimiento descrito en la Resolución CONAMA No. 382/2006 de la legislación brasileña, que establece los límites máximos de emisión de contaminantes atmosféricos para fuentes fijas, es decir, los que son emitidos por los hornos y calderas de las empresas industriales.
Los equipos que se utilizaron para la recolección de los gases de combustión fueron la Colección isocinética de contaminantes atmosféricos - CIPA fabricado por TECNAL y el analizador de gases de combustión fabricado por Instrutemp. Todos los equipos fueron calibrados de acuerdo con la Red Brasileña de Calibración - RBC.

Todas las empresas encuestadas tienen una potencia térmica nominal (MW) de los hornos entre $>0,15 \mathrm{y} \leq 1,0$ por lo que los límites establecidos por la legislación para esta potencia son los descritos en la tabla 2 según la potencia del horno.

Tabla 2 - Límites establecidos para fuentes fijas estacionarias hornos de calcinación.

\begin{tabular}{|c|c|c|c|}
\hline $\begin{array}{c}\mathrm{CO}=1.700 \\
\mathbf{m g} / \mathbf{N}_{\mathbf{N}} \mathbf{3}\end{array}$ & $\begin{array}{c}\text { NOx }=\text { No se } \\
\text { aplica }\end{array}$ & $\begin{array}{c}\mathrm{MP}=730 \\
\mathbf{m g} / \mathbf{N} . \mathbf{m}^{\mathbf{3}}\end{array}$ & $\begin{array}{c}\mathrm{SOx}=2.700 \\
\mathbf{m g} / \mathbf{N} \cdot \mathbf{m}^{\mathbf{3}}\end{array}$ \\
\hline
\end{tabular}

Fuente: Autores.

\section{Resultados y Discusión}

En el municipio de Trindade se realizaron mediciones en nueve empresas, siete de las cuales utilizan leña como combustible, una utiliza coque y la otra emplea carbón en sus dos hornos. Las pruebas se realizaron en todas las empresas en 2015 y se repitieron en los años siguientes 2016, 2017 y 2018 para que poder emitir una comparación de los resultados de las mediciones de contaminantes atmosféricos. La Tabla 3 describe los resultados encontrados para los hornos de las 9 empresas analizadas en el municipio de Trindade.

Tabla 3 - Resultados de las mediciones de contaminantes atmosféricos en el municipio de Trindade (2015 - 2016).

\begin{tabular}{|c|c|c|c|c|c|c|c|c|c|}
\hline \multirow{3}{*}{ Empresa } & \multicolumn{4}{|c|}{2015} & \multicolumn{5}{|c|}{2016} \\
\hline & $\mathrm{CO}$ & NOx & $\mathrm{SOx}$ & MP & $\mathrm{CO}$ & NOx & $\mathrm{SOx}$ & MP & Combustible \\
\hline & \multicolumn{4}{|c|}{$\mathrm{mg} / \mathrm{N} \cdot \mathrm{m}^{3}$} & \multicolumn{5}{|c|}{$\mathrm{mg} / \mathrm{N} \cdot \mathrm{m}^{3}$} \\
\hline Empresa 1 & 4.845 & 102 & & 427 & 227,2 & 63,4 & & 123 & Leña \\
\hline Empresa 2 & 5.309 & 115 & 183 & 1.023 & 989 & 214 & 162 & 693 & Coque \\
\hline Empresa 3 & 79,9 & 106 & & 1.380 & 3.597 & 6,25 & & 489,3 & Leña \\
\hline Empresa 4 & 537 & 57,4 & & 178 & 25.585 & 36,1 & & 794 & Leña \\
\hline Empresa 5 & 587 & 59,7 & & 198 & 234 & 124 & & 697 & Leña \\
\hline Empresa 6 & 129 & 649 & & 532 & 2.305 & 37,9 & & 678 & Leña \\
\hline Empresa 7 & 1.892 & 5,4 & & 893 & 8.852 & 1,26 & & 568 & Leña \\
\hline Empresa 8.1 & 1.532 & 144 & 7.840 & 148 & 9,608 & 1,47 & 247 & 1.345 & Carbón \\
\hline \begin{tabular}{|l|} 
Empresa 8.2 \\
\end{tabular} & 937 & 97,2 & 457 & 2.134 & 4.641 & 154 & 189 & 894,2 & Carbón \\
\hline Empresa 9 & 1.922 & 30,8 & & 931 & 1.345 & 87,9 & & 684 & Leña \\
\hline
\end{tabular}

Nota: Todos los resultados que se destacan enfatizados muestran valores superiores a los permitidos por la legislación vigente, Consejo Nacional del Medio Ambiente - CONAMA.

Fuente: Autores.

En el período 2015 y 2016 en la ciudad de Trindade, la empresa 2 utilizó coque de petróleo y la empresa 8 utilizó carbón mineral en dos hornos, por eso se identificaron como 8.1 y 8.2, las otras empresas utilizaron leña como combustible.

En cuanto a los resultados presentados en la Tabla 3, se encontró que el 89\% de las empresas encuestadas en 2015 y 2016 emiten contaminantes a la atmósfera fuera de los estándares establecidos por la Resolución CONAMA No. 382 de 2006.

De acuerdo con la Figura 2 se puede verificar que las empresas 1, 2, 7 y 9 superaron el límite máximo permitido de emisión de monóxido de carbono - CO, mientras que la empresa 8.1 que utiliza carbón mineral superó el límite de óxidos de azufre - SOx, en comparación con la Resolución CONAMA n 382 de 2006. 
Figura 2 - Emisiones contaminantes al municipio de Trindade en los años 2015 y 2016.

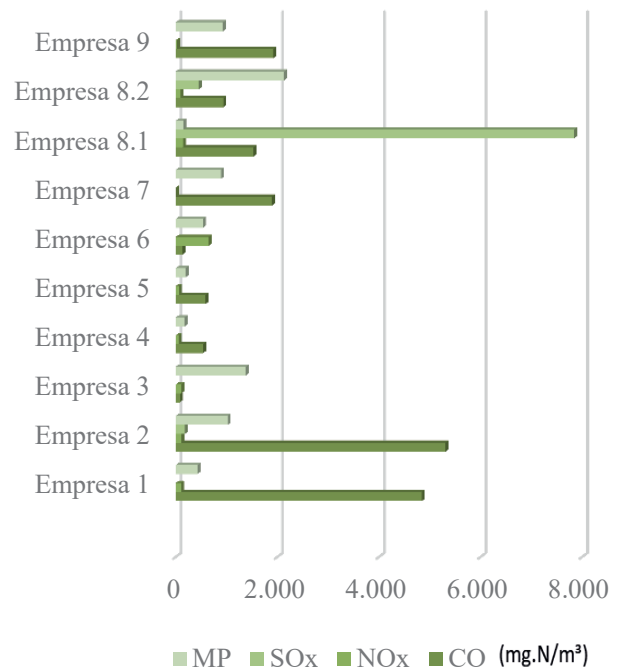

Fuente: Autores.

En relación con los resultados presentados en 2015 y 2016 , se observa que algunas empresas siguen superando los límites de emisiones contaminantes. La mayoría de las empresas encuestadas en 2015 no tenían una licencia de operación de la agencia ambiental, pero se estaban regularizando. En 2016, todas las empresas ya contaban con una licencia de operación, aunque un año después de que los resultados de las pruebas estuvieran fuera de los estándares establecidos por la legislación, algunas empresas continuaron emitiendo

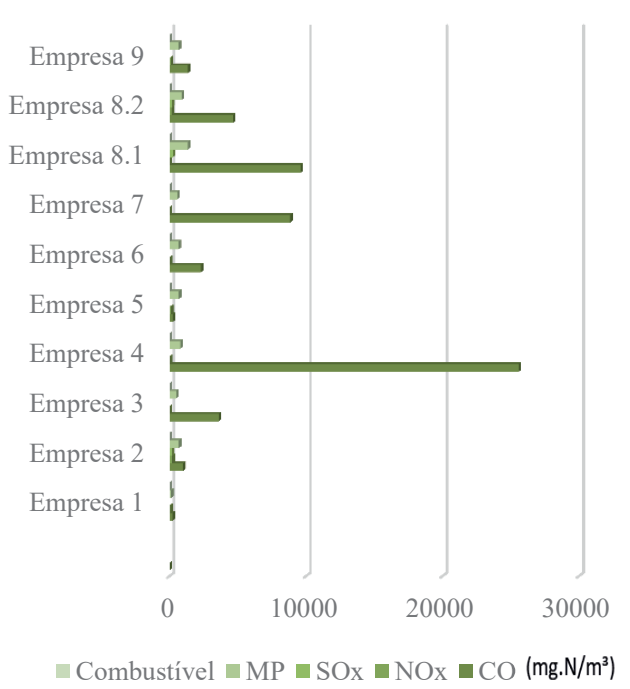

contaminantes a la atmósfera por encima de lo permitido.

En 2017 y 2018, en la ciudad de Trindade, las empresas 2 y 8 que utilizaban otros combustibles fósiles hicieron modificaciones en sus hornos y comenzaron a utilizar leña como combustible.

Se verificó que el $100 \%$ de las empresas encuestadas en 2017 y 2018 emiten contaminantes a la atmósfera fuera de los estándares establecidos por la Resolución CONAMA No. 382 de 2006 como se observa en la Tabla 4.

Tabla 4 - Resultados de las mediciones de contaminantes atmosféricos en el municipio de Trindade (2017 - 2018)

\begin{tabular}{|c|c|c|c|c|c|c|c|}
\hline \multicolumn{4}{|c|}{2017} & \multicolumn{4}{|c|}{2018} \\
\hline Empresa & $\mathrm{CO}$ & NOx & MP & CO & NOx & MP & Combustible \\
\hline \multicolumn{4}{|c|}{$\mathrm{mg} / \mathrm{N} \cdot \mathrm{m}^{3}$} & \multicolumn{4}{|c|}{$\mathrm{mg} / \mathrm{N} \cdot \mathrm{m}^{3}$} \\
\hline Empresa 1 & 7.962 & 4,48 & 287,1 & 3.102 & 157 & 674,2 & Leña \\
\hline Empresa 2 & 2.347 & 153,1 & 783,4 & 1.237 & 243,6 & 213,1 & Leña \\
\hline Empresa 3 & 11.282 & 16,9 & 156,2 & 5.337 & 0 & 544,7 & Leña \\
\hline Empresa 4 & 13.993 & 47,1 & 789,0 & 5.990 & 54,1 & 435,6 & Leña \\
\hline Empresa 5 & 10.568 & 27,6 & 942,5 & 7.990 & 265,3 & 53,33 & Leña \\
\hline Empresa 6 & 6.060 & 19,8 & 763,6 & 8.485 & 174,9 & 742,9 & Leña \\
\hline Empresa 7 & 8.470 & 9,9 & 435,1 & 1.771 & 332,9 & 845,0 & Leña \\
\hline Empresa 8.1 & 2.503 & 15,8 & 132,9 & 6.759 & 15,4 & 951,3 & Leña \\
\hline Empresa 8.2 & 3.256 & 25,3 & 287,1 & 2.009 & 101,4 & 241,0 & Leña \\
\hline Empresa 9 & 2.354 & 128 & 794,2 & 793 & 389,9 & 385,3 & Leña \\
\hline
\end{tabular}

Fuente: Autores.

En la Figura 3 se observa que en 2017 el 100\% de las empresas superaron los límites de emisión de monóxido de carbono - CO y el 50\% superó el límite de emisión de partículas - PM. Se incrementó en un 40\% el número de empresas que han superado los límites de la legislación en relación con el contaminante $\mathrm{CO}$, por lo que el control y seguimiento de la agencia ambiental de estas empresas no se está llevando a cabo de manera satisfactoria para cumplir con la legislación pertinente.

De la misma forma para el año 2018 sólo las empresas 
2 y 9 presentaron resultados satisfactorios para todos los parámetros analizados. En relación con las partículas, sólo las empresas 7 y 8.1 presentaron resultados superiores a los permitidos por la legislación.

Figura 3 - Emisión de contaminantes a la ciudad de Trindade durante 2017 y 2018.

2017

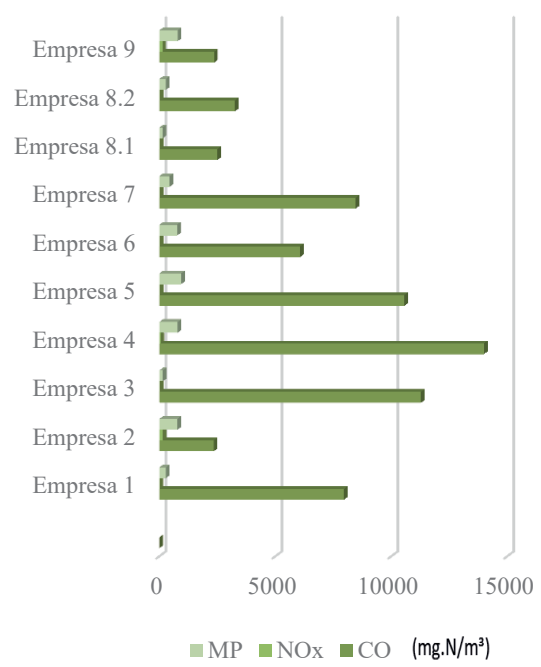

Fuente: Autores
2018

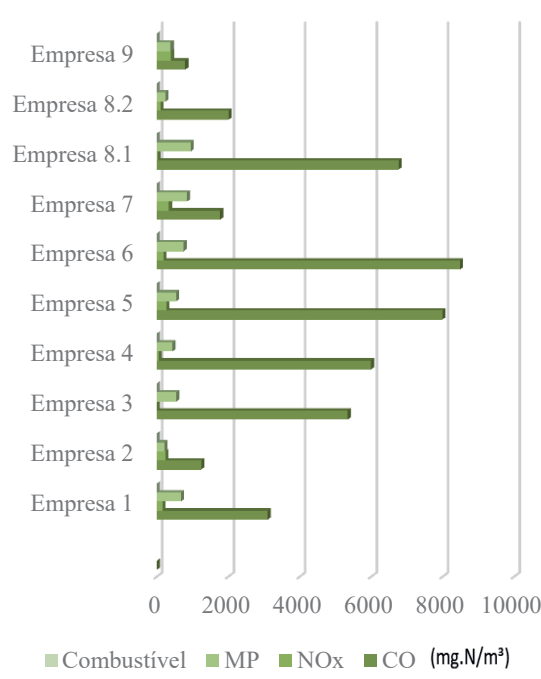

De acuerdo con la investigación realizada, del análisis derivado de las mediciones a 11 empresas, todas utilizan

Tabla 5 - Resultados de las mediciones de contaminantes atmosféricos en el municipio de Araripina (2015 - 2016).

\begin{tabular}{|c|c|c|c|c|c|c|c|}
\hline \multirow{3}{*}{ Empresa } & \multicolumn{3}{|c|}{2015} & \multicolumn{4}{|c|}{2016} \\
\hline & $\mathrm{CO}$ & NOx & MP & $\mathrm{CO}$ & NOx & MP & Combustible \\
\hline & \multicolumn{3}{|c|}{ mg.N/m ${ }^{3}$} & \multicolumn{4}{|c|}{ mg.N/m ${ }^{3}$} \\
\hline Empresa 1 & 38,5 & 6,16 & 1.399 & 2.234 & 144,9 & 123 & Leña \\
\hline Empresa 2 & 298,3 & 0,93 & 652 & 3.159 & 22,6 & 329 & Leña \\
\hline Empresa 3 & 3.798 & 83,6 & 1.235 & 7.709 & 0,9 & 238 & Leña \\
\hline Empresa 4 & 453,8 & 137,3 & 1.127 & 2.191 & 128 & 432 & Leña \\
\hline Empresa 5 & 3.191 & 47,3 & 2.543 & 420,3 & 66,3 & 576 & Leña \\
\hline Empresa 6 & 7.223 & 87,5 & 1.893 & 347 & 131 & 643 & Leña \\
\hline Empresa 7 & 5.871 & 72,3 & 1.325 & 2.846 & 117,4 & 159 & Leña \\
\hline Empresa 8 & 4.009 & 56,2 & 2.335 & 6.797 & 52,2 & 320 & Leña \\
\hline Empresa 9 & 6.721 & 125,3 & 897 & 1.699 & 14,3 & 105 & Leña \\
\hline Empresa 10 & 6.098 & 87,4 & 945 & 5.443 & 193,6 & 698 & Leña \\
\hline Empresa 11 & 4.987 & 59,1 & 782 & 8.750 & 21,0 & 298 & Leña \\
\hline
\end{tabular}

Fuente: Autores.

Cada año de acuerdo a los contaminantes atmosféricos presentados, se encontró que en el año 2015 un total del 90\% de las empresas emitieron contaminantes por encima de lo permitido por la legislación, en el año 2016 hubo una reducción al $73 \%$ de las empresas que emitieron contaminantes por encima de lo determinado por la Resolución CONAMA No. 382 de 2006.

De acuerdo con la Figura 4, en 2015 el 90\% de las empresas emitió material particulado por encima del nivel permitido y en 2016 el 100\% de las empresas no superó el límite máximo de emisión permitido para material particulado en comparación con la Resolución CONAMA No. 382 de 2006.

Para los años 2017 y 2018, el 90\% de las empresas encuestadas en 2017 y el $63 \%$ de las empresas encuestadas en 2018 cumplieron con la legislación en relación a las emisiones 
de material particulado - PM, pero en relación al monóxido de carbono el $73 \%$ continúa emitiendo contaminantes a la atmósfera fuera de los estándares establecidos por la legislación de la Resolución CONAMA No. 382 de 2006.

Figura 4 - Emisión de contaminantes en el municipio de Araripina para los años 2015 y 2016.

2015

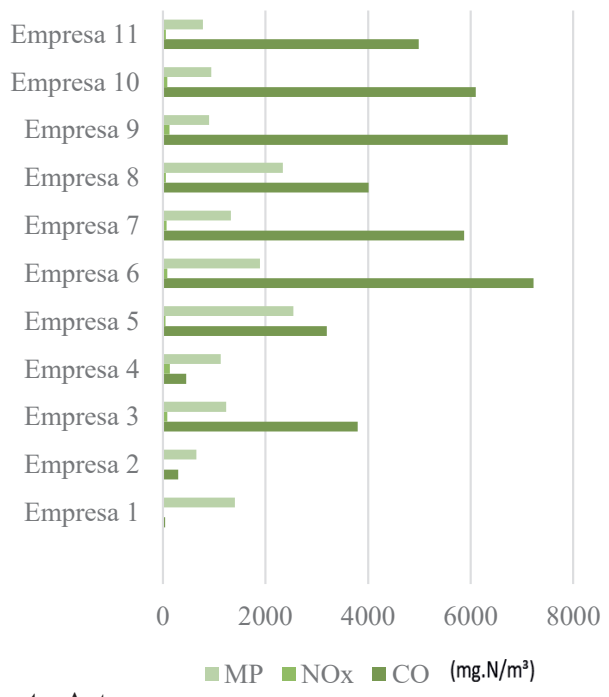

2016

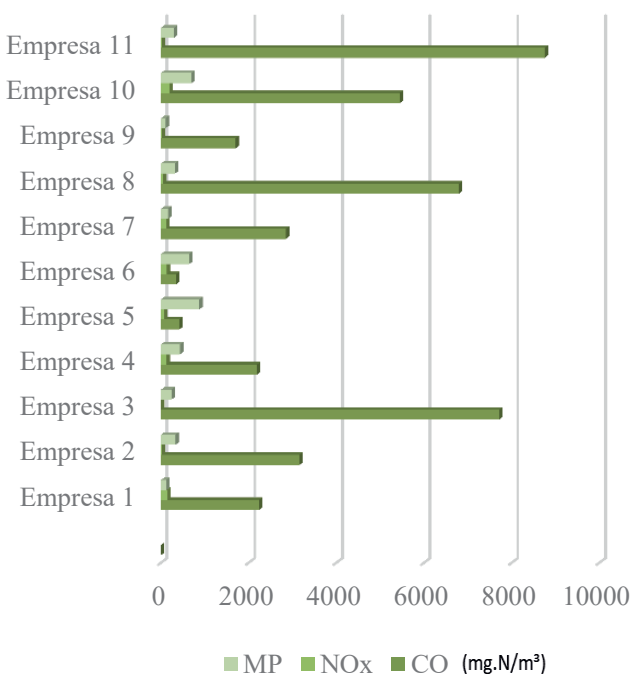

Tabla 6 - Resultados de las mediciones de contaminantes atmosféricos en el municipio de Araripina (2017 - 2018)

\begin{tabular}{|c|c|c|c|c|c|c|c|}
\hline \multirow{3}{*}{ Empresa } & \multicolumn{3}{|c|}{2017} & \multicolumn{4}{|c|}{2018} \\
\hline & $\mathrm{CO}$ & NOx & MP & $\mathrm{CO}$ & NOx & MP & Combustible \\
\hline & \multicolumn{3}{|c|}{ mg.N/m ${ }^{3}$} & \multicolumn{4}{|c|}{$\mathrm{mg} \cdot \mathrm{N} / \mathrm{m}^{3}$} \\
\hline Empresa 1 & 7.962 & 4,48 & 287 & 2.137 & 4,3 & 432 & Leña \\
\hline Empresa 2 & 2.347 & 153 & 783 & 16.720 & 177 & 146 & Leña \\
\hline Empresa 3 & 11.282 & 16,9 & 156 & 2.046 & 325 & 354 & Leña \\
\hline Empresa 4 & 13.993 & 47,1 & 789 & 18.763 & 348 & 398 & Leña \\
\hline Empresa 5 & 10.568 & 27,6 & 942 & 4.464 & 36,6 & 268 & Leña \\
\hline Empresa 6 & 6.060 & 19,8 & 763 & 2.423 & 54,2 & 583 & Leña \\
\hline Empresa 7 & 8.470 & 9,9 & 435 & 1.479 & 67,3 & 69,2 & Leña \\
\hline Empresa 8 & 2.503 & 15,8 & 132 & 985 & 82,7 & 593 & Leña \\
\hline Empresa 9 & 1.345 & 25,6 & 569 & 2.349 & 59,2 & 623 & Leña \\
\hline Empresa 10 & 3.452 & 68,7 & 645 & 1.856 & 127 & 524 & Leña \\
\hline Empresa 11 & 4.962 & 120 & 891 & 987 & 231 & 418 & Leña \\
\hline
\end{tabular}

Fuente: Autores.

Se analizó también que para los años 2017 y 2018, el control de las emisiones de monóxido de carbono alcanza niveles hasta 10 veces superiores a los permitidos por la legislación, y el control y seguimiento de estas empresas no se está llevando a cabo de forma satisfactoria para cumplir con la legislación pertinente, como se observa en la Figura 5.

\section{Conclusion}

Del 2015 al 2018 no hubo una reducción significativa de las emisiones contaminantes atmosféricas en los hornos de las empresas encuestadas en los municipios de Araripina y Trindade, al no haber inversiones para mejorar el proceso de quema y mantenimiento de los hornos utilizados.

La matriz energética utilizada entre 2015 y 2016 fue coque y carbón, sustituyéndose en el 2017 con derivados de la madera para eliminar la emisión de óxidos de azufre a la atmósfera.

Las emisiones de monóxido de carbono excedieron el límite permitido por la legislación ambiental hasta en 10 veces. La gran mayoría de las empresas son micro y pequeñas y los hornos se encuentran en condiciones precarias que requieren mantenimiento y mejora en el proceso de combustión, lo que resulta en una combustión incompleta que aumenta la emisión de contaminantes atmosféricos. 
Figura 5 - Emisión de contaminantes en el municipio de Araripina para los años 2017 y 2018.

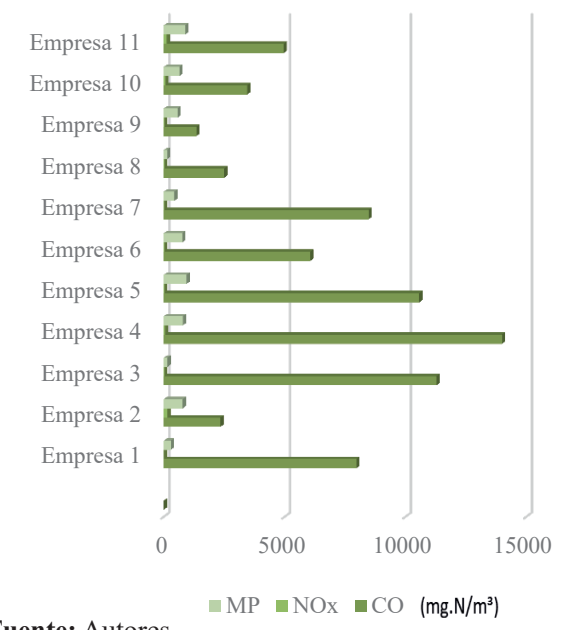

Fuente: Autores.

A partir de 2016, las empresas del municipio de Araripina redujeron la contaminación por partículas para mantener las emisiones debajo de lo permitido por la legislación en los años siguientes hasta 2018, mediante el uso de sistemas de escape para la retención del material particulado.

Con el aumento de la inspección de los organismos ambientales, las empresas de calcinación de yeso de la Región de Araripe están utilizando como combustible leña inspeccionada y autorizada, de la gestión forestal. Esto garantiza la conservación de la catinga, como vegetación natural de la región semiárida del nordeste brasileño.

\section{Referencias}

ABNT - Associação Brasileira de Normas Técnicas. NBR 10701/1989 - Determinação de pontos de amostragem em dutos e chaminés de fontes estacionárias. Rio de Janeiro. 1989.

ABNT - Associação Brasileira de Normas Técnicas. NBR 11966/1989 - Efluentes gasosos em dutos e chaminés de fontes estacionárias - ABNT - Associação Brasileira de Normas Técnicas Determinação da velocidade e vazão. Rio de janeiro. 1989.

ABNT - Associação Brasileira de Normas Técnicas. NBR 11967/1989 - Efluentes gasosos em dutos e chaminés de fontes estacionárias - Determinação da umidade. Rio de janeiro. 1989.

BARBISAN, A.O. et al. Impactos ambientais causados pela construção civil. Unoesc \& Ciência-ACSA, v. 2, n. 2, p. 173-180, 2011.

BRAGA, A.L.F.; ZANOBETTI, A.; SCHWARTZ, J. The lag structure between particulate air pollution and respiratory and cardiovascular deaths in ten US cities. J. Occup. Environ. Med., v.43, n.11, p.927-933, 2001.

BRASIL. Ministério da Saúde. Fundação Nacional de Saúde. Curso básico de vigilância ambiental em saúde (CBVA). Brasília: MS, 2001.

CBIC - Câmara Brasileira da Indústria da Construção. Relatório Técnico. 2018. Disponible en: https://cbic.org.br/publicacoes. Acesso: 20 jul. 2019.

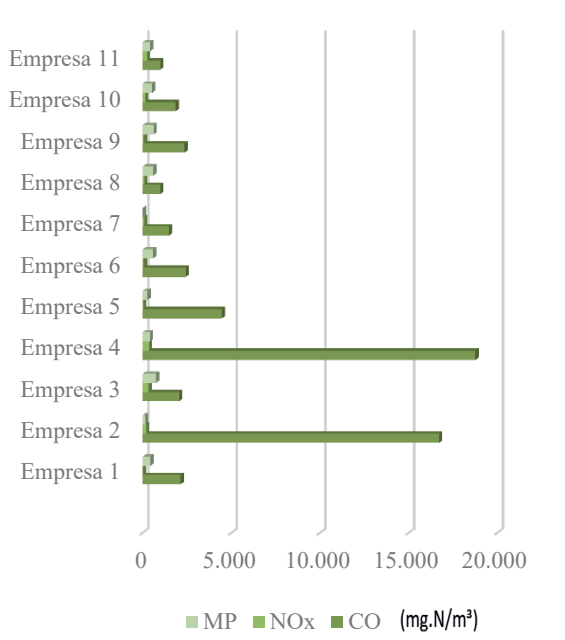

CANÇADO, J.E.D. et al. Repercussões clínicas da exposição à poluição atmosférica. J. Bras. Pneumol., v. 32, n. Supl. 1, p. S5S11, 2006.

CARVALHO, C.M. et al. Análise dos gases de exaustão de chaminés com o uso do aparelho de orsat. J. Eng., Tecnol. Meio Amb., v.2, n.2, p.7-12, 2017.

CASTRO, A.H.; SILVA, G.M.; ARAÚJO, R.S. Qualidade do ar-parâmetros de controle e efeitos na saúde humana: uma breve revisão. Holos, v.5, p.107-121, 2013.

CONAMA. Resolução Conama n. ${ }^{\circ}$ 382. 2006. Disponible en: http://www.mma.gov.br/port/conama/legiabre.cfm? codlegi=520. Acceso: 30 jul.2019.

DOCKERY, D.W. et al. An association between air pollution and mortality in six US cities. N. Engl. J. Med., v. 329, p. 1753-1759, 1993.

DRUMM, F. C. et al. Poluição atmosférica proveniente da queima de combustíveis derivados do petróleo em veículos automotores. Rev. Eletr. Gestão, Educ. Tecnol. Amb., v.18, n.1, p.66-78, 2014.

EISINGER, M.; BURROWS, J. P. Tropospheric sulfur dioxide observed by the ERS $\square 2$ GOME instrument. Geophysical Res. Letters, v.25, n.22, p.4177-4180, 1998.

FELLENBERG, G. Introdução aos problemas da poluição ambiental. São Paulo: E.P.U./EDUSP. 1997.

GALAN, I. et al. Short-term effects of air pollution on daily asthma emergency room admissions. Euro. Respirat. J., v.22, n.5, p.802-808, 2003.

GASQUES, A.C. Impactos ambientais dos materiais da construção civil: breve revisão teórica. Rev. Tecnol., v. 23, n.1, p.13-24, 2015.

GONÇALVES, A.B.R.; NASCIMENTO, D.A. Favela, espaço e sujeito: uma relação conflituosa. Rev. Ipotesi, v.15, n.2, p.51-62. 2011.

GURGEL, L.V.A.; GIL, L.F. Adsorption of Cu (II), Cd (II) and $\mathrm{Pb}$ (II) from aqueous single metal solutions by succinylated twice-mercerized sugarcane bagasse functionalized with triethylenetetramine. Water Res., v.43, n.18, p.4479-4488, 2009. doi: 10.1016/j.watres.2009.07.017 
JARDIM, W.F. A evolução da atmosfera terrestre. 2001. Disponible en: http://qnesc.sbq.org.br/online/cadernos/01/ evolucao.pdf. Acceso: 20 sep.2019.

JOHN, V. M.; CINCOTTO, M. A. Alternativas de gestão dos resíduos de gesso. São Paulo: Universidade de São Paulo, 2003.

LUSHNIKOVA, N.; DVORKIN, L. Sustainability of gypsum products as a construction material. In: KHATIB, J. (Ed.). Sustainability of Construction Materials. Woodhead Publishing, 2016. p.643-681.

MEDEIROS, M. S. Poluição ambiental por exposição à poeira de gesso: impactos na saúde da população. 2003. Disponible en: https://www.cpqam.fiocruz.br/bibpdf/2003medeiros-ms.pdf. Acceso: 25 sep.2019.

MEDEIROS, M. S.; HURTADO-GUERRERO, J.C.; SILVA, L.G.A. Health in the context of the gypsum production area of the city of Araripina, State of Pernambuco, Brazil. Saúde Soc., v.19, n.2, p.358-370, 2010.

MIRAGLIA, S.G.E.K.; GOUVEIA, N. Custos da poluição atmosférica nas regiões metropolitanas brasileiras. Ciência \& Saúde Coletiva, v. 19, p. 4141-4147, 2014. doi: 10.1590/1413812320141910.09232014

MOUVIER G. A poluição atmosférica. Lisboa: Biblioteca Básica de Ciência e Cultura - Instituto Piaget, 1995.

OIT - Oficina Internacional Del Trabajo. Yeso. Enciclopedia de salud y seguridad en el trabajo. Madrid: Ministerio del Trabajo y Seguridad Social, 1989.

PARENTE, A.H. Modelagem e simulação do efeito de parede na transmissão de calor em um forno rotativo utilizado na produção de gesso beta. 2001. Disponible en: http://repositorio.unicamp.br/ bitstream/REPOSIP/267582/1/Parente_AntonioHelder_D.pdf. Acceso: 12 sep.2019.
PINHEIRO, S.M.M. Gesso reciclado: avaliação de propriedades para uso em componentes. 2011. Disponible en: http://repositorio. unicamp.br/jspui/handle/REPOSIP/257820. Acesso: 31 ago. 2019.

PROCKOP, L.D.; CHICHKOVA, R.I. Carbon monoxide intoxication: an updated review. J. Neurol. Scie., v. 262, n. 1/2, p.122-130, 2007. doi: 10.1016/j.jns.2007.06.037

SANTOS, F. M. M. Análise das emissões de poluentes atmosféricos de fontes fixas industriais em Portugal continental. 2006. Disponible en: https://repositorium.sdum.uminho. pt/bitstream/1822/7572/1/Art.\%203\%209th\%20Conf.\%20 Nacional\%20Ambiente\%20Abril2007.pdf. Acceso: 23 sep.2019.

SANTOS, M. V.; SARDOU, R. Diagnóstico das atividades econômicas do pólo gesseiro do Araripe. Recife: SEBRAE, 1996.

SEVERO, E.M. F. Estruturação de Modelo para Avaliação dos Riscos Decorrentes da Exposição do Trabalhador à Poeira do Gesso. In: CONGRESSO IBERO-AMERICANO EM INVESTIGAÇÃO QUALITATIVA EM SAÚDE. 2017, Salamanca. Anais. Salamanca: CIAIQ, 2017, p. 1136-1144.

USEPA - United States Environmental Protection Agency. Industrial Guide for Air Pollution Control, Technology Transfer Washington, DC EPA 625/6-78-004. 1978.

USEPA - United States Environmental Protection Agency. Provisional Assessment of Recent Studies on Health Effects of Particulate Matter Exposure. 2012. Disponível em: http://cfpub. epa.gov/ncea $/$ isa/recordisplay.cfm?d eid=247132. Acceso: 31 ago. 2019.

YANAGI, Y.; ASSUNÇÃO, V. J.; BARROZO, V. L. Influência do material particulado atmosférico na incidência e mortalidade por câncer no Município de São Paulo, Brasil. Cad. Saúde Pública, v.28, n.9, p.1737-1748, 2012. 